\title{
Bianchi type I cosmologies in arbitrary dimensional dilaton gravities
}

\author{
Chiang-Mei Chen* \\ Department of Physics, National Central University, Chungli 320, Taiwan \\ T. Harko ${ }^{\dagger}$ \\ Department of Physics, University of Hong Kong, Pokfulam Road, Hong Kong \\ M. K. Mak \\ Department of Physics, Hong Kong University of Science and Technology, Clear Water Bay, Hong Kong
}

(Received 17 April 2000; published 22 November 2000)

\begin{abstract}
We study the low energy string effective action with an exponential type dilaton potential and vanishing torsion in a Bianchi type I space-time geometry. In the Einstein and string frames the general solution of the gravitational field equations can be expressed in an exact parametric form. Depending on the values of the dilaton coupling constant and of the coefficient in the exponential, the obtained cosmological models can be generically divided into three classes, leading to both singular and non-singular behaviors. The effect of the potential on the time evolution of the mean anisotropy parameter is also considered in detail, and it is shown that a Bianchi type I universe isotropizes only in the presence of a dilaton field potential or a central deficit charge.

PACS number(s): 04.20.Jb, 04.65.+e, 98.80.-k
\end{abstract}

\section{INTRODUCTION}

In an attempt to address the potential, inherited from string theory, to eliminate the initial cosmological singularity, from which time and our Universe are supposed to have begun about $15 \times 10^{9}$ years ago, Gasperini and Veneziano initiated a program known as the pre-big-bang scenario [1]. The field equations of the pre-big-bang cosmology are based on the low energy effective action resulting from string theory. In $D$ dimensions, the massless bosonic fields from the Neveu-Schwarz-Neveu-Schwarz (NS-NS) sector are the dilaton $\phi$, the antisymmetric tensor $B_{\mu \nu}$, and the metric tensor $\hat{g}_{\mu \nu}$, whose dynamics is described, in the "string frame," by the following action [2-4]:

$$
\hat{S}=\int d^{D} x \sqrt{-\hat{g}} e^{-2 \phi}\left\{\hat{R}+\hat{\kappa}(\hat{\nabla} \phi)^{2}-\frac{1}{12} H_{[3]}^{2}-\hat{U}(\phi)\right\},
$$

where $H_{[3]}=d B_{[2]}$ and $\hat{\kappa}$ is a generalized dilaton coupling constant ( $\hat{\kappa}=4$ for superstring theories). Moreover, we also allow for the existence of a potential $\hat{U}(\phi)$ of the dilaton field.

From a physical point of view the most important candidate for the potential is a cosmological constant $\Lambda$, which appears in the massive extension of type IIA supergravity and is restricted to be positive, $\Lambda>0$ [4]. The field equations derived from the string effective action admit inflationary solutions that are driven by the kinetic energy associated with the massless fields rather than any interaction potential.

\footnotetext{
*Email address: cmchen@joule.phy.ncu.edu.tw

†Email address: tcharko@hkusua.hku.hk

‡Email address: mkmak@vtc.edu.hk
}

There are many massless fields present in the pre-big-bang scenario, such as the dilaton, graviton and moduli fields. For an extensive recent review of string cosmology see [5].

For simplicity in the following we assume that $H_{[3]}$ is vanishing. In this circumstance, via a conformal rescaling

$$
g_{\mu \nu}=e^{[-4 /(D-2)] \phi} \hat{g}_{\mu \nu}
$$

the action (1) reduces to a $D$-dimensional dilaton gravity whose action, in the "Einstein frame," has the form

$$
S=\int d^{D} x \sqrt{-g}\left\{R-\kappa(\nabla \phi)^{2}-U(\phi)\right\},
$$

with $U(\phi)=e^{4 \phi /(D-2)} \hat{U}(\phi)$ and $\kappa=4(D-1) /(D-2)-\hat{\kappa}$.

Pre-big-bang inflationary cosmological models, based on the action (1) or (3), have been recently intensively investigated in the physical literature [6-16]. Gasperini and Ricci [6] have obtained exact solutions to the four-dimensional low energy string effective action adopting a spaceindependent dilaton and vanishing Kalb-Ramond antisymmetric tensor field ansatz for the Bianchi type I, II, III, $\mathrm{V}, \mathrm{VI}_{0}$ and $\mathrm{VI}_{h}$ geometries. They have shown that in such a context the initial curvature singularities cannot be avoided. Brandenberger, Easther and Maia [7] have found nonsingular spatially homogeneous and isotropic solutions for dilaton gravity in the presence of a special combination of higher derivative terms in the gravitational action. Some of these solutions correspond to a spatially flat, bouncing universe originating in a dilaton-dominated contracting phase and emerging as an expanding Friedmann-Robertson-Walker (FRW) universe.

A general framework for studying large classes of cosmological solutions of the low-energy limit of type II string theory and of $\mathrm{M}$ theory, for space-time decomposable into a set of flat or maximally symmetric spatial subspaces, with 
non-trivial Ramond form fields excited, has been developed by Lukas, Ovrut and Waldram [14]. In their formalism the low energy equations of motion are equivalent to those describing a particle moving in a moduli space consisting of the scale factors of the subspaces together with the dilaton. Two classes of exact solutions have been presented, corresponding to exciting only a single form and with multiple forms excited. The resulting exact solutions begin and end in a curvature singularity.

Very recently, the string cosmology equations with a dilaton potential have been examined, in the string frame, by Ellis et al. [15], who also give a generic algorithm for obtaining solutions with desired evolutionary properties. The presence of a dilaton potential leads to the violation of the pre-big-bang symmetry $a(t) \rightarrow 1 / a(t)$. Moreover, Garcia de Andrade [16] obtained several classes of solutions of the Einstein-Cartan dilatonic inflationary cosmology. In the cases where the dilatons are constrained by the presence of spin-torsion effects a repulsive gravity is found. The temperature fluctuation has also been computed from the nearly flat spectrum of the gravitational waves produced during inflation, with results agreeing with the Cosmic Background Explorer (COBE) data.

Pre-big-bang cosmological models, in which there is no need to introduce the inflation or to fine-tune potentials, have many attractive features [17]. Inflation is natural, thanks to the duality symmetries of string cosmology, and the initial condition problem is decoupled from the singularity problem. Finally, quantum instability (pair creation) is able to heat up an initially cold universe and generate a standard hot big bang with the additional features of homogeneity, flatness and isotropy.

The cosmological behavior of universes filled with a scalar field, $\phi$, as well as a Liouville type exponential potential, has been extensively investigated in the physical literature for both homogeneous and inhomogeneous scalar fields [1833] (for a summary see [33]). Scalar fields are considered to play a central role in current models of the early Universe. The self-interaction potential energy density of such a field is undiluted by the expansion of the Universe and hence can act like an effective cosmological constant driving a period of inflation. The evolution of the Universe is strongly dependent upon the specific form of the scalar field potential $U$. A common form for the self-interaction potential is the exponential type potential. An exponential potential arises in four-dimensional effective Kaluza-Klein type theories from compactification of the higher-dimensional supergravity or superstring theories [2]. In string or Kaluza-Klein theories the moduli fields associated with the geometry of the extra dimensions may have effective exponential potentials due to the curvature of the internal spaces or to the interaction of the moduli with form fields on the internal spaces. Exponential potentials can also arise due to non-perturbative effects such as gaugino condensation [34]. In the Einstein frame the exponential potential is also generated by means of the conformal transformation (2) for $\hat{U}(\phi)=\Lambda$, with $\Lambda$ the central charge deficit.

A solution in the case of a flat space-time filled with a scalar field with an exponential potential but describing power-law inflationary behavior has been obtained by Barrow [18]. Homogeneous and inhomogeneous fourdimensional Bianchi type I space-time in the presence of scalar fields with exponential potential have been studied in $[21,22]$. Higher dimensional $(D \geqslant 4)$ anisotropic cosmological models with a massless scalar field self-interacting through an exponential potential have been investigated in [26]. A non-inflationary solution for an open FRW universe exponential-potential pure scalar field filled space-time and with scalar field energy density decaying as $\rho_{\phi} \sim t^{-2}$ has been recently found by Mubarak and Oezer [29].

It is the purpose of the present paper to study Bianchi type I cosmological models in the dilaton gravity (1) and (3). More specifically, we consider the effects of a Liouville type exponential potential, $U(\phi)=U_{0} e^{\lambda \phi}$, with arbitrary values of the constants $U_{0}, \lambda$, on the dynamics and evolution of an anisotropic space-time, in both the Einstein and string frames.

In the case of the dilaton field self-interacting through an exponential potential the general solution of the gravitational field equations in a flat Bianchi type I geometry can be expressed in an exact parametric form in both Einstein and string frames. The effects of the potential on the evolution of the basic physical parameters (mean anisotropy, shear and deceleration) of the anisotropic space-time are also considered in detail.

The present paper is organized as follows. The basic equations describing the dilatonic Bianchi type I cosmological model are obtained in Sec. II. The general solution of the field equations for an exponential type dilaton potential is obtained in Sec. III (Einstein frame) and in Sec. IV (string frame). In Sec. V we discuss our results and conclusions.

\section{GEOMETRY, EINSTEIN FRAME FIELD EQUATIONS AND CONSEQUENCES}

In this paper, we shall consider the $D$-dimensional anisotropic generalization of the flat FRW geometry-the Bianchi type I space-time-described by the line element

$$
d s^{2}=-d t^{2}+\sum_{i=1}^{D-1} a_{i}^{2}(t)\left(d x^{i}\right)^{2} .
$$

For this metric, it is convenient to introduce the following variables: volume scale factor $V$, directional Hubble factors $H_{i}$ and mean Hubble factor $H$ as

$$
\begin{aligned}
V & :=\prod_{i=1}^{D-1} a_{i}, \\
H_{i} & :=\frac{\dot{a}_{i}}{a_{i}}, \quad i=1, \ldots, D-1, \\
H & :=\frac{1}{D-1} \sum_{i=1}^{D-1} H_{i}, \\
\Delta H_{i} & :=H_{i}-H, \quad i=1, \ldots, D-1 .
\end{aligned}
$$


Then one can immediately check out the relation

$$
H=\frac{1}{D-1} \frac{\dot{V}}{V} \text {. }
$$

In terms of variables $(5)-(8)$ the Ricci tensor of the Bianchi type I geometry can be expressed as

$$
\begin{aligned}
R_{00} & =-\frac{d}{d t} \sum_{i=1}^{D-1}\left(\frac{\dot{a}_{i}}{a_{i}}\right)-\sum_{i=1}^{D-1}\left(\frac{\dot{a}_{i}}{a_{i}}\right)^{2} \\
& =-(D-1) \dot{H}-\sum_{i=1}^{D-1} H_{i}^{2}, \\
R_{i i} & =a_{i}^{2}\left[\dot{H}_{i}+(D-1) H H_{i}\right], \quad i=1, \ldots, D-1 .
\end{aligned}
$$

On the other hand, the field equations of the action (3) can be achieved by variation with respect to the fields $g^{\mu \nu}$ and $\phi$, giving

$$
\begin{array}{r}
R_{\mu \nu}-\kappa \nabla_{\mu} \phi \nabla_{\nu} \phi-\frac{U}{D-2} g_{\mu \nu}=0, \\
\nabla^{2} \phi-\frac{1}{2 \kappa} \frac{\partial U}{\partial \phi}=0,
\end{array}
$$

where $\nabla$ is the covariant derivative of $g_{\mu \nu}$. Thus, for the Bianchi type I space-time, the gravitational field equations in the Einstein frame reduce to

$$
\begin{gathered}
(D-1) \dot{H}+\sum_{i=1}^{D-1} H_{i}^{2}+\kappa \dot{\phi}^{2}-\frac{1}{D-2} U=0, \\
\frac{1}{V} \frac{d}{d t}\left(V H_{i}\right)-\frac{1}{D-2} U=0, \quad i=1, \ldots, D-1, \\
\frac{1}{V} \frac{d}{d t}(V \dot{\phi})+\frac{1}{2 \kappa} \frac{\partial U}{\partial \phi}=0 .
\end{gathered}
$$

By summing Eqs. (15) we obtain

$$
\frac{1}{V} \frac{d}{d t}(V H)=\frac{1}{D-2} U,
$$

which, together with Eq. (15), leads to

$$
H_{i}=H+\frac{K_{i}}{V}, \quad i=1, \ldots, D-1
$$

In Eqs. (18), $K_{i}, i=1, \ldots, D-1$, are constants of integration, which satisfy the relation:

$$
\sum_{i=1}^{D-1} K_{i}=0
$$

Substituting Eqs. (18) into Eq. (14) and then combining with Eq. (17) we obtain

$$
\kappa \dot{\phi}^{2}+(D-2) \dot{H}+\frac{K^{2}}{V^{2}}=0
$$

where $K^{2}:=\sum_{i=1}^{D-1} K_{i}^{2}$. Consequently, the remaining task is to solve Eqs. (16), (17) and (20).

The physical quantities of interest in cosmology are the expansion scalar $\theta$, the mean anisotropy parameter $A$, the shear scalar $\Sigma^{2}$ and the deceleration parameter $q$ and are defined according to [35]

$$
\begin{aligned}
\theta:=(D-1) H=\frac{\dot{V}}{V} \\
A:=\frac{1}{D-1} \sum_{i=1}^{D-1}\left(\frac{\Delta H_{i}}{H}\right)^{2}=\frac{1}{D-1} \frac{K^{2}}{V^{2} H^{2}}, \\
\Sigma^{2}:=\frac{1}{D-2}\left(\sum_{i=1}^{D-1} H_{i}^{2}-(D-1) H^{2}\right)=\frac{D-1}{D-2} A H^{2}, \\
q:=\frac{d}{d t} H^{-1}-1 .
\end{aligned}
$$

The sign of the deceleration parameter indicates whether the cosmological model inflates. The positive sign corresponds to standard decelerating models whereas the negative sign indicates inflationary behavior.

\section{EXPONENTIAL POTENTIAL IN THE EINSTEIN FRAME}

The cosmological dynamics and evolution of the dilaton field filled Bianchi type I universe essentially depends on the functional form of the potential $U(\phi)$. In the present paper we restrict our study to the exponential type potential,

$$
U(\phi)=U_{0} e^{\lambda \phi},
$$

with $U_{0}$ and $\lambda$ arbitrary constants. For this type of potential, the combination of Eqs. (16) and (17) leads to

$$
\frac{d}{d t}\left(\frac{1}{D-2} V \dot{\phi}+\frac{\lambda}{2 \kappa} V H\right)=0
$$

or, equivalently, to

$$
\dot{\phi}=\frac{(D-2) C}{V}-\frac{(D-2) \lambda}{2 \kappa} H
$$

with $C$ a constant of integration.

Substitution of Eq. (27) into Eq. (20) gives the "final", field equation

$$
\frac{\ddot{V}}{V}+\alpha \frac{\dot{V}^{2}}{V^{2}}-\beta \frac{\dot{V}}{V^{2}}+\gamma \frac{1}{V^{2}}=0,
$$

where 


$$
\begin{aligned}
& \alpha=\frac{(D-2) \lambda^{2}}{4(D-1) \kappa}-1, \\
& \beta=(D-2) C \lambda, \\
& \gamma=(D-1)(D-2) C^{2} \kappa+\frac{(D-1) K^{2}}{D-2} .
\end{aligned}
$$

By introducing a new variable $u:=\dot{V}$, Eq. (28) takes the form

$$
\frac{u d u}{-\alpha u^{2}+\beta u-\gamma}=\frac{d V}{V} .
$$

Equation (32) has the general solution (with $V_{0}$ a constant of integration)

$$
V=V_{0} \exp \left(\int \frac{u d u}{-\alpha u^{2}+\beta u-\gamma}\right)
$$

In the following we denote

$$
\begin{aligned}
& \Delta=\beta^{2}-4 \alpha \gamma, \\
& u_{0}=\frac{\beta}{2 \alpha}, \\
& u_{ \pm}=\frac{\beta \pm \sqrt{\Delta}}{2 \alpha}, \\
& m_{ \pm}=-\frac{1}{2 \alpha}\left(1 \pm \frac{\beta}{\sqrt{\Delta}}\right) .
\end{aligned}
$$

Hence, taking $u$ as a parameter, we obtain three classes of solutions of the gravitational field equations describing a dilaton field filled Bianchi type I pre-big-bang universe. The explicit form of the solutions depends on the values of the parameters $\alpha, \beta$ and $\gamma$. All the solutions are expressed in a closed parametric form and are given by the following:

(A) $\Delta>0$ :

$$
\begin{aligned}
& t=t_{0}-\frac{V_{0}}{\alpha} \int\left(u-u_{+}\right)^{m_{+}-1}\left(u-u_{-}\right)^{m_{-}-1} d u, \\
& V=V_{0}\left(u-u_{+}\right)^{m+}\left(u-u_{-}\right)^{m_{-}}, \\
& a_{i}=a_{i 0} \prod_{\epsilon= \pm} u^{-K_{i} m_{\epsilon} / u_{\epsilon}}\left(u-u_{\epsilon}\right)^{\left[1 /(D-1)+K_{i} / u_{\epsilon}\right] m_{\epsilon}}, \\
& i=1, \ldots, D-1 \text {, } \\
& q=(D-2)+\frac{(D-1) \alpha}{u^{2}} \prod_{\epsilon= \pm}\left(u-u_{\epsilon}\right), \\
& U=-\frac{(D-2) \alpha}{(D-1) V_{0}^{2}} \prod_{\epsilon= \pm}\left(u-u_{\epsilon}\right)^{1-2 m_{\epsilon}} .
\end{aligned}
$$

(B) $\Delta=0$ :

$$
\begin{gathered}
t=t_{0}-\frac{V_{0}}{\alpha} \int \frac{\exp \left(\frac{u_{0}}{\alpha\left(u-u_{0}\right)}\right)}{\left(u-u_{0}\right)^{1 / \alpha+2}} d u, \\
V=V_{0}\left(u-u_{0}\right)^{-1 / \alpha} \exp \left(\frac{u_{0}}{\alpha\left(u-u_{0}\right)}\right),
\end{gathered}
$$

$$
\begin{array}{r}
a_{i}=a_{i 0}\left(u-u_{0}\right)^{-1 /(D-1) \alpha} \exp \left(\frac{u_{0}+K_{i}(D-1)}{\alpha(D-1)\left(u-u_{0}\right)}\right) \\
i=1, \ldots, D-1,
\end{array}
$$

$$
q=(D-2)+\frac{(D-1) \alpha\left(u-u_{0}\right)^{2}}{u^{2}},
$$

$$
U=-\frac{(D-2) \alpha}{(D-1) V_{0}^{2}}\left(u-u_{0}\right)^{2 / \alpha+2} \exp \left(\frac{-2 u_{0}}{\alpha\left(u-u_{0}\right)}\right)
$$

(C) $\Delta<0$ :

$$
\begin{aligned}
t= & t_{0}+V_{0} \int\left(-\alpha u^{2}+\beta u-\gamma\right)^{-1 / 2 \alpha-1} \\
& \times \exp \left(-\frac{\beta}{\alpha \sqrt{-\Delta}} \arctan \frac{2 \alpha u-\beta}{\sqrt{-\Delta}}\right) d u \\
V= & V_{0}\left(-\alpha u^{2}+\beta u-\gamma\right)^{-1 / 2 \alpha} \\
& \times \exp \left(-\frac{\beta}{\alpha \sqrt{-\Delta}} \arctan \frac{2 \alpha u-\beta}{\sqrt{-\Delta}}\right) \\
a_{i}= & a_{i 0}\left(-\alpha u^{2}+\beta u-\gamma\right)^{-1 / 2 \alpha(D-1)} \\
& \times \exp \left(-\frac{\beta+2 \alpha K_{i}(D-1)}{\alpha(D-1) \sqrt{-\Delta}} \arctan \frac{2 \alpha u-\beta}{\sqrt{-\Delta}}\right), \\
& \times \exp \left(\frac{2 \beta}{\alpha \sqrt{-\Delta}}\right) \\
& \frac{\left.D-2 \arctan \frac{2 \alpha u-\beta}{\sqrt{-\Delta}}\right)}{(D-1) V_{0}^{2}\left(-\alpha u^{2}+\beta u-\gamma\right)^{1 / \alpha+1}} \\
q= & (D-2)-(D-1) \frac{-\alpha u^{2}+\beta u-\gamma}{u^{2}}
\end{aligned}
$$

For all three cases and with the use of definitions given by Eqs. (21)-(23) the quantities $\theta, A$ and $\Sigma^{2}$ can be found from 


$$
\theta=\frac{u}{V}, \quad A=\frac{(D-1) K^{2}}{u^{2}}, \quad \Sigma^{2}=\frac{K^{2}}{(D-2) V^{2}}
$$

The general solution of the gravitational field equations for a dilatonic Bianchi type I universe with exponential potential given by Eqs. (38)-(53) is valid only for a particular range of the parameters $u$ and $\alpha$. These ranges follow from the conditions of the positivity of the potential $U(\phi)$ and of the reality of basic physical quantities such as, for example, the scale factors. The positivity of the potential $U(\phi)$ $>0, \forall t \geqslant t_{0}$ implies $\alpha<0$ in all three cases (A), (B) and (C). The expressions for the physical parameters make sense only for values of the parameter $u$ so that $u>u_{+}, u_{-}$in case (A) and $u>u_{0}$ in case (B) [in case (C) one requires again $\alpha<0$ ].

The value of the constant $U_{0}$ in the potential (25) is not arbitrary, but it is determined in terms of the parameters of the solution.

\section{EXPONENTIAL POTENTIAL IN THE STRING FRAME}

In the string frame the gravitational field equations and the dilaton equations are obtained by varying the action (1) and, under the assumption of vanishing $H_{[3]}$, are given by

$$
\begin{array}{r}
\hat{R}_{\mu \nu}-\frac{1}{2} \hat{g}_{\mu \nu} \hat{R}+2 \hat{\nabla}_{\mu} \hat{\nabla}_{\nu} \phi+(\hat{\kappa}-4) \hat{\nabla}_{\mu} \phi \hat{\nabla}_{\nu} \phi \\
-\frac{1}{2} \hat{g}_{\mu \nu}\left\{4 \hat{\nabla}^{2} \phi+(\hat{\kappa}-8)(\hat{\nabla} \phi)^{2}-\hat{U}\right\}=0, \\
\hat{R}+\hat{\kappa} \hat{\nabla}^{2} \phi-\hat{\kappa}(\hat{\nabla} \phi)^{2}-\hat{U}(\phi)+\frac{1}{2} \frac{\partial \hat{U}}{\partial \phi}=0 .
\end{array}
$$

By eliminating $\hat{R}$ between Eqs. (54) and (55), the gravitational field and dilaton equations take the form

$$
\begin{aligned}
\hat{R}_{\mu \nu}+2 \hat{\nabla}_{\mu} \hat{\nabla}_{\nu} \phi+(\hat{\kappa}-4) \hat{\nabla}_{\mu} \phi \hat{\nabla}_{\nu} \phi-\frac{\hat{g}_{\mu \nu}}{2}\left\{(4-\hat{\kappa}) \hat{\nabla}^{2} \phi\right. \\
\left.\quad+2(\hat{\kappa}-4)(\hat{\nabla} \phi)^{2}-\frac{1}{2} \frac{\partial \hat{U}}{\partial \phi}\right\}=0 \\
\hat{\nabla}^{2} \phi-2(\hat{\nabla} \phi)^{2}+\frac{4 \hat{U}+(D-2) \frac{\partial \hat{U}}{\partial \phi}}{2[(D-2) \hat{\kappa}-4(D-1)]}=0
\end{aligned}
$$

In the present section we consider the general solution of Eqs. (54) and (55) for an exponential type potential, $\hat{U}(\phi)$ $=\hat{U}_{0} \exp (\hat{\lambda} \phi)$, with $\hat{\lambda}$ an arbitrary constant. Since the metric tensors are connected via the conformal transformation (2), in the string frame the general solutions of the gravitational field equations can be obtained by applying the conformal transformation (2) to the solution obtained in the Einstein frame. In the string frame we also assume an anisotropic Bianchi type I geometry with line element

$$
d \hat{s}^{2}=-d \hat{t}^{2}+\sum_{i=1}^{D-1} \hat{a}_{i}^{2}(\hat{t})\left(d x^{i}\right)^{2},
$$

with the metric tensor components in the two frames connected by the conformal transformation (2) and with the time coordinate $\hat{t}$ defined according to

$$
\hat{t}=\int \exp \left[\frac{2}{D-2} \phi(t)\right] d t
$$

In the two frames the volume scale factor, the directional Hubble factors and the mean Hubble factor are related by means of the general relations

$$
\begin{aligned}
\hat{V} & =V e^{[2(D-1) / D-2] \phi}, \\
\hat{H}_{i} & =\left(H_{i}+\frac{2}{D-2} \dot{\phi}\right) e^{-[2 /(D-2)] \phi}, \quad i=1, \ldots, D-1, \\
\hat{H} & =\left(H+\frac{2}{D-2} \dot{\phi}\right) e^{-[2 /(D-2)] \phi} .
\end{aligned}
$$

To apply the conformal transformation, we need first to find the conformal transformation factor $e^{\phi}$. With the use of Eq. (21) we find

$$
\begin{aligned}
\frac{1}{V} \frac{d}{d t}(V H) & =\frac{1}{D-1} \frac{1}{V} \frac{d \dot{V}}{d t} \\
& =\frac{1}{D-1} \frac{1}{V} \frac{d u}{d t} \\
& =\frac{1}{D-1} \frac{1}{V} \frac{d u}{d V} \frac{d V}{d t} \\
& =\frac{u}{(D-1) V^{2}} \frac{d u}{d \ln V}
\end{aligned}
$$

Then from Eq. (17) it is easy to obtain that the potential $U(\phi)$ can be expressed as

$$
U(\phi)=\frac{D-2}{D-1} \frac{u}{V^{2}}\left(\frac{d \ln V}{d u}\right)^{-1},
$$

leading to

$$
e^{\phi}=\left[\frac{D-2}{(D-1) U_{0}}\right]^{1 / \lambda}\left(\frac{V^{2}}{u} \frac{d \ln V}{d u}\right)^{-1 / \lambda} .
$$

Therefore in the string frame the general solution of the gravitational field equation for a dilaton field filled Bianchi type I with an exponential potential of the form

$$
\hat{U}(\phi)=U_{0} \exp \left[\left(\lambda-\frac{4}{D-2}\right) \phi\right]
$$


with $\lambda$ an arbitrary constant, can be expressed again in an exact closed parametric form, with $u$ taken as parameter, and is given by

$$
\begin{aligned}
& \hat{t}-\hat{t}_{0}=\left[\frac{D-2}{(D-1) U_{0}}\right]^{2 /(D-2) \lambda} \int\left(\frac{V^{2}}{u} \frac{d \ln V}{d u}\right)^{1-2 /(D-2) \lambda} \frac{d u}{V}, \\
& \hat{V}=V\left[\frac{D-2}{(D-1) U_{0}}\right]^{2(D-1) /(D-2) \lambda} \\
& \times\left(\frac{V^{2}}{u} \frac{d \ln V}{d u}\right)^{-2(D-1) /(D-2) \lambda}, \\
& \hat{H}=\left[\frac{D-2}{(D-1) U_{0}}\right]^{-2 /(D-2) \lambda}\left(\frac{V^{2}}{u} \frac{d \ln V}{d u}\right)^{2 /(D-2) \lambda} \\
& \times\left[\frac{2 C}{V}+\frac{\kappa-\lambda}{(D-1) \kappa} \frac{u}{V}\right], \\
& \hat{a}_{i}=a_{i 0}\left[\frac{D-2}{(D-1) U_{0}}\right]^{2 /(D-2) \lambda}\left(\frac{V^{2}}{u} \frac{d \ln V}{d u}\right)^{-2 /(D-2) \lambda} \\
& \times V^{1 /(D-1)} \exp \left(K_{i} \int \frac{1}{u} \frac{d \ln V}{d u} d u\right), \\
& i=1, \ldots, D-1 \text {, } \\
& \hat{A}=(D-1) \sum_{i=1}^{D-1}\left[\frac{\kappa K_{i}}{(\kappa-\lambda) u+2 \kappa C(D-1)}\right]^{2}, \\
& \hat{q}=(D-2)-\frac{u}{D-1}\left(\frac{d \ln V}{d u}\right)^{-1}\left[2 C+\frac{\kappa-\lambda}{(D-1) \kappa} u\right]^{-2}, \\
& \hat{U}=U_{0}\left[\frac{D-2}{(D-1) U_{0}}\right]^{1-4 /(D-2) \lambda}\left(\frac{V^{2}}{u} \frac{d \ln V}{d u}\right)^{4 /(D-2) \lambda-1} .
\end{aligned}
$$

In the string frame there are also three distinct classes of solutions, corresponding to $\Delta>0, \Delta=0$ and $\Delta<0$ respectively. Substituting the values of $V$ obtained in the previous section in the formulas given above, we can find, via straightforward calculations, the explicit parametric representations, for each class of solutions, of the general solution of the gravitational field equations for a dilaton field filled Bianchi type I space-time, with an arbitrary exponential potential.

If in the solution given above we take $\lambda=4 /(D-2)$, we obtain the general solution of the gravitational field equations in the string frame corresponding to a constant potential or, equivalently, to a cosmological constant. In this case also there are three distinct classes of solutions, with all physical quantities represented as exact functions of time. For $\hat{U}(\phi)$ $\equiv \Lambda=$ const, Eq. (66) becomes

$$
\hat{t}-\hat{t}_{0}=\sqrt{\frac{D-2}{(D-1) \Lambda}} \int \frac{d u}{\sqrt{-\alpha u^{2}+\beta u-\gamma}} .
$$

In order to obtain solutions defined for all values of the parameters we shall assume in the following that $\alpha<0$. Then Eq. (73) has the solutions

$$
\begin{aligned}
& u=-\frac{\beta}{2|\alpha|}+\delta_{+} \cosh \frac{\hat{t}-\hat{t}_{0}}{\hat{\tau}_{0}}, \text { for } \Delta>0, \\
& u=-\frac{\beta}{2|\alpha|}+\exp \left(\frac{\hat{t}-\hat{t}_{0}}{\hat{\tau}_{0}}\right), \text { for } \Delta=0, \\
& u=-\frac{\beta}{2|\alpha|}+\delta_{-} \sinh \frac{\hat{t}-\hat{t}_{0}}{\hat{\tau}_{0}}, \text { for } \Delta<0,
\end{aligned}
$$

where we denoted $\delta_{ \pm}:=\sqrt{ \pm \Delta} / 2|\alpha|$ and $\hat{\tau}_{0}$ $:=\sqrt{D-2 /(D-1) \Lambda|\alpha|}$.

In this way we can obtain the exact (non-parametric) solution for the anisotropic Bianchi type I geometry in the presence of a central charge deficit. We shall not present here the resulting formulas, due to their complicated (but elementary) mathematical form. As compared to the Einstein frame, the evolution in the string frame of the Universe in the presence of the cosmological constant can be quite complicated.

\section{DISCUSSIONS AND FINAL REMARKS}

In order to consider the general effects of a dilaton field potential in the Einstein frame on the dynamics and evolution of an arbitrary dimensional Bianchi type I space-time, we also give the general solution of the gravitational field equations (14)-(16) corresponding to $U(\phi) \equiv 0$. The corresponding Kasner-type solutions are not new (for an extensive review of this type of string cosmological models see [5]). For the case of the zero potential we immediately obtain

$$
\begin{aligned}
V & =V_{0} t, \\
H & =\frac{1}{(D-1) t}, \\
a_{i} & =a_{i 0} t^{p_{i}}, \quad i=1, \ldots, D-1, \\
A & =\frac{(D-1) K^{2}}{V_{0}^{2}}=\text { const } \\
q & =D-2=\text { const, } \\
\phi & =\phi_{0} \ln t,
\end{aligned}
$$

where

$$
\phi_{0}=\sqrt{\frac{1}{\kappa}\left(\frac{D-2}{D-1}-\frac{K^{2}}{V_{0}^{2}}\right)},
$$

and an arbitrary constant of integration that can be added to Eq. (82) has been taken to be 1 . The coefficients $p_{i}:=1 /(D$ 
$-1)+K_{i} / V_{0}$ satisfy the relations $\sum_{i=1}^{D-1} p_{i}=1$ and $\sum_{i=1}^{D-1} p_{i}^{2}$ $=1 /(D-1)+K^{2} / V_{0}^{2}$. Hence in the Einstein frame the geometry of the potential free dilaton field is of Kasner type, but with $\sum_{i=1}^{D-1} p_{i}^{2} \neq 1$ (if we adopt the normalization $\sum_{i=1}^{D-1} p_{i}^{2}$ $=1$, then we obtain the empty Bianchi type I universe with $\phi \equiv 0)$. The anisotropic Bianchi type I dilaton field filled universe does not isotropize (the mean anisotropy parameter is a constant for all times) and its evolution is noninflationary with $q>0$ for all $t$.

In order to analyze the general effects of the dilaton field potential on the dilaton field filled Bianchi type I space-time in the Einstein frame, we obtain first the following anisotropy equation:

$$
\frac{d A}{d t}=-\frac{2 A}{H}\left[\dot{H}+(D-1) H^{2}\right],
$$

which can also be written in the equivalent form

$$
\frac{d A}{d t}=-\frac{2 A U(\phi)}{(D-2) H}
$$

and integrated to give

$$
A(t)=A_{0} \exp \left(-\frac{2}{D-2} \int_{t_{0}}^{t} \frac{U(\phi)}{H} d t\right) .
$$

In Eq. (85) we denoted by $A_{0}$ an arbitrary constant of integration. For $U(\phi) \equiv 0$ we always have $A \equiv A_{0}=$ const. If $\int_{t_{0}}^{t}[U(\phi) / H] d t$ is a monotonically increasing positive function of time, then the presence of the dilaton field potential will lead to the fast isotropization of the Bianchi type I space-time.

In the presence of a potential the deceleration parameter $q=-\left(\dot{H}+H^{2}\right) / H^{2}$ can be expressed as

$$
q=(D-2)-\frac{U(\phi)}{(D-2) H^{2}} .
$$

If $U(\phi) \equiv 0$ in the Einstein frame, the evolution of the Universe is non-inflationary, but once the condition $U(\phi)>(D$ $-2)^{2} H^{2}$ is satisfied, the dynamics of the Bianchi type I space-time becomes inflationary.

In the present paper we have obtained the general solution of the gravitational field equations for a Bianchi type I spacetime filled with a dilaton field with an exponential potential in both the Einstein and string frame. In the Einstein frame they describe generically an expanding universe, with $u=\dot{V}$ $\geqslant 0$ and with properties strongly dependent on the numerical values of the physical parameters describing the dilaton field and its potential. A contracting universe with $u=\dot{V}<0$ generally does not satisfy the condition of reality of the scale factors. The solutions of the field equations can be classified into three classes, according to the sign of the quantity $\Delta$. In the limit of large $u, u \rightarrow \infty$, all three solutions have a similar behavior. The mean anisotropy $A$ tends in all cases to zero, indicating that an exponential type potential leads to the isotropization of the Universe.
In four space-time dimensions, as shown in [21], the physical properties of the solutions of the gravitational field equations for a Bianchi type I space-time filled with an exponential potential scalar field are determined by the coefficient $\lambda$ in the exponential. For $\lambda^{2}<6$ (and with $\kappa=1$ ) one has flat, isotropic power law solutions which are attractors for all expanding solutions [21]. The $D$-dimensional generalization of this conditions follows from the restriction $\alpha$ $<0$ imposed to assure the positivity of the potential and is given by

$$
\lambda^{2}<\frac{4(D-1) \kappa}{D-2}
$$

In the large $u$ limit the deceleration parameter behaves as $q=(D-2)+\alpha(D-1)$. If the condition $\alpha<-(D-2) /(D$ $-1)$ or, equivalently,

$$
\lambda^{2}<\frac{4 \kappa}{D-2}
$$

is fulfilled, the Universe will enter in an inflationary phase. In four dimensions and with $\kappa=1$ the solutions inflate for $\lambda^{2}<2$ [21]. For values of $\alpha$ which do not satisfy this condition the evolution of the space-time will be generally noninflationary. In the same limit of large $u$ the scalar field is given by $\phi \sim[(\alpha+1) / \alpha] \ln u$.

Multi-dimensional ( $D>4$ ) Kasner type (non-isotropizing) solutions in string and M-theory cosmology, with either an initial or a final curvature singularity were considered recently in [14]. In the case of compactifications on maximally symmetric subspaces the moduli-space potential, which arises when non-trivial form fields are excited, significantly affects the structure of the solution. The potential in [14] is written in a more systematic way as $U$ $=\frac{1}{2} \sum_{r=1}^{m} u_{r}^{2} \exp \left(q_{r I} \alpha_{I}\right)$, where the sums run over all elementary and solitonic configurations as well as a possible cosmological term. $u_{r}$ represents integration constants. The type of each term is specified by vectors $q_{r}, r=1, \ldots, m$. In the language of Lukas, Ovrut and Waldram [14] the condition $\alpha<0$ is the condition that the vector $q$ be timelike. In the paper [14] the authors always considered $q$ spacelike, thus leading to $\alpha>0$ and, consequently, with solutions always asymptoting to a Kasner-like anisotropic geometry. But we must also note that typical string models actually give the regime $\alpha>0$ [14].

For class (A) solutions, the Bianchi type I dilaton field filled universe starts in the Einstein frame from a singular state, corresponding to the values $u=u_{+}$or $u=u_{-}$of the parameter. Hence for this model a singular state with zero values of the scale factors is unavoidable. But for class (B) of solutions the evolution of the Universe is non-singular for $u_{0}<0$. In this case the scale factors are finite for all finite values of the parameter $u$. Alternatively, class (C) models are non-singular for values of the constants $\alpha$ and $\gamma$ such that $\alpha<0$ and $\gamma<0$.

In Figs. 1-4 we have represented the variations in the Einstein frame of the volume scale factor, mean anisotropy, deceleration parameter and dilaton field for a four- 


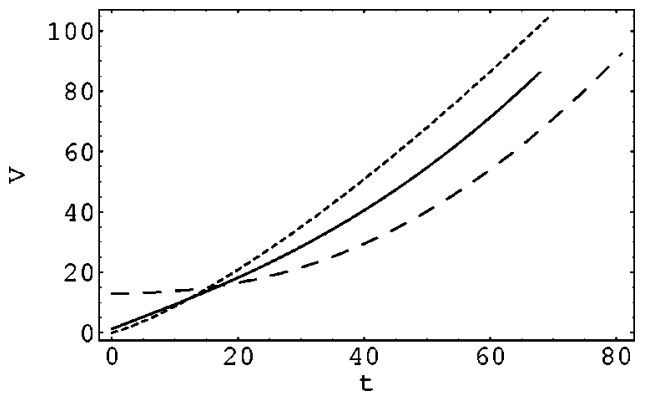

FIG. 1. Time evolution in the Einstein frame of the fourdimensional $(D=4)$ volume scale factor $V(t)$ of the dilaton field filled Bianchi type I universe with exponential potential for different values of the parameters $\alpha, \beta$ and $\gamma$ : (i) Class (A) model (solid curve), $\alpha=-\frac{1}{3}, \quad \beta=1, \quad \gamma=1$. (ii) Class (B) model (dotted curve), $u_{0}=1$. (iii) Class (C) model (dashed curve), $\alpha=-\frac{1}{3}, \beta=1, \gamma$ $=-1$.

dimensional $(D=4)$ Bianchi type I space-time. The anisotropic universe will always end in an isotropic state, but its dynamics can be either inflationary or non-inflationary. Generally the dilaton field $\phi$ is a decreasing function of time.

We shall consider now the effects of the dilaton field and potential on the dynamics and evolution of a Bianchi type I space-time in the string frame. In the case in which there is no dilaton field potential, $\hat{U}(\phi) \equiv 0$, the general solution of the gravitational field equations and of the dilaton equation can be obtained again by the conformal transformation (2) from Eqs. (77)-(82). Hence in this case we obtain first the relation connecting the time coordinate in the string and Einstein frames in the form

$$
t=\left(\frac{\hat{t}}{\hat{n}}\right)^{\hat{n}}
$$

where

$$
\hat{n}=\frac{D-2}{D-2+2 \phi_{0}} \text {. }
$$

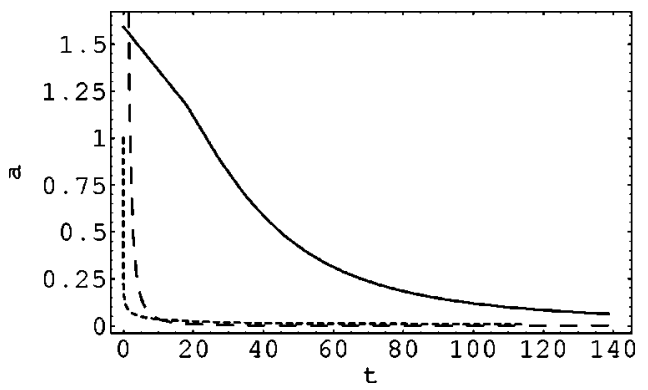

FIG. 2. Einstein frame time evolution of the four-dimensional mean anisotropy parameter $a(t):=A(t) / 3 K^{2}$ of the dilaton field filled Bianchi type I universe with exponential potential for different values of the parameters $\alpha, \beta$ and $\gamma$ : (i) Class (A) model (solid curve), $\alpha=-\frac{1}{3}, \quad \beta=1, \gamma=1$. (ii) Class (B) model (dotted curve), $u_{0}=1$. (iii) Class (C) model (dashed curve), $\alpha=-\frac{1}{3}, \beta=1, \gamma=$ -1 . An expanding Bianchi type I universe always isotropizes in the presence of an exponential dilaton potential.

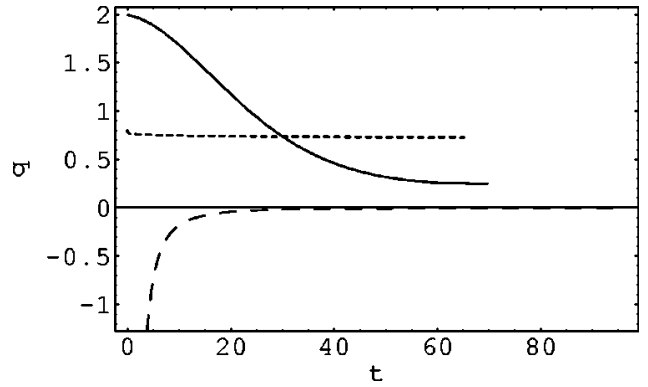

FIG. 3. Dynamics of the four-dimensional $(D=4)$ deceleration parameter $q(t)$ of the dilaton field filled Bianchi type I universe with exponential potential, in the Einstein frame, for different values of the parameters $\alpha, \beta$ and $\gamma$ : (i). Class (A) model (solid curve), $\alpha=-\frac{1}{3}, \beta=1, \gamma=1$. (ii) Class (B) model (dotted curve), $u_{0}=1$. (iii) Class (C) model (dashed curve), $\alpha=-\frac{1}{3}, \beta=1, \gamma=$ -1 . Depending on the values of the parameters the Bianchi type universe has both inflationary and non-inflationary evolutions.

In the string frame the general solution of the potential free dilaton field filled anisotropic universe is given by

$$
\begin{aligned}
& \hat{V}=\hat{V}_{0} \hat{t}^{\hat{h}}, \\
& \hat{H}=\frac{\hat{h}}{(D-1) \hat{t}}, \\
& \hat{a}_{i}=\hat{a}_{i 0} \hat{t}^{\hat{p}_{i}}, \quad i=1, \ldots, D-1,
\end{aligned}
$$

and

$$
\hat{A}:=\frac{1}{D-1} \sum_{i=1}^{D-1}\left(\frac{\Delta \hat{H}_{i}}{\hat{H}}\right)^{2}=\frac{1}{D-1} \sum_{i=1}^{D-1}\left[1-\frac{(D-1) \hat{p}_{i}}{\hat{h}}\right]^{2},
$$

$$
\hat{q}=\frac{D-1}{\hat{h}}-1
$$

where $\hat{V}_{0}$ and $\hat{a}_{i 0}$ are arbitrary constants of integration. Here we also denoted

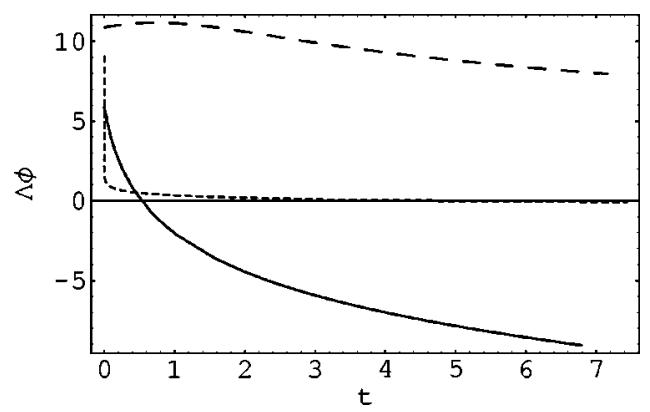

FIG. 4. Variation in the Einstein frame of the four-dimensional $(D=4)$ dilaton field $\phi(t)$ for different values of the parameters $\alpha, \beta$ and $\gamma$. (i) Class (A) model (solid curve), $\alpha=-\frac{1}{3}, \beta=1, \gamma$ $=1$. (ii) Class (B) model (dotted curve), $u_{0}=1$. (iii) Class (C) model (dashed curve), $\alpha=-\frac{1}{3}, \beta=1, \gamma=-1$. 


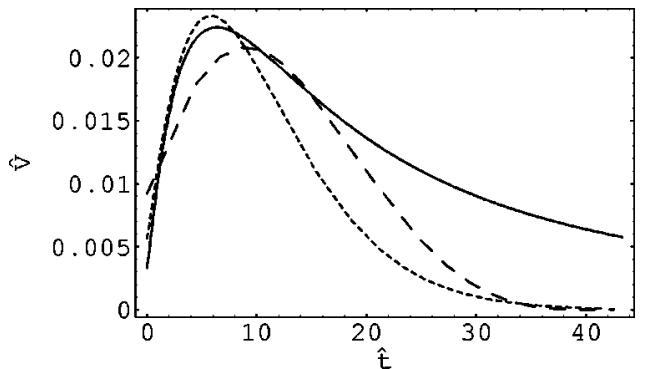

FIG. 5. String frame evolution of the volume scale factor $\hat{V}$ of the dilatonic Bianchi type I universe in the presence of an exponential potential $\hat{U}=U_{0} \exp \{[\lambda-4 /(D-2)] \phi\}$ as a function of time $\hat{t}$ for $\alpha=-1 / 3, \beta=1, \gamma=1$ and for different values of $\lambda: \quad \lambda=3$ (solid curve), $\lambda=2$ (this case corresponds to the presence of a central charge deficit or cosmological constant) (dotted curve) and $\lambda$ $=1$ (dashed curve). We have used the normalization $(D-2) /(D$ -1) $U_{0}=1$.

$$
\begin{aligned}
\hat{h} & =\frac{D-2+2(D-1) \phi_{0}}{D-2+2 \phi_{0}}, \\
\hat{p}_{i} & =\hat{n}\left(p_{i}+\frac{2 \phi_{0}}{D-2}\right), \quad i=1, \ldots, D-1,
\end{aligned}
$$

and the coefficients $\hat{p}_{i}$ satisfy the relations

$$
\begin{aligned}
& \sum_{i=1}^{D-1} \hat{p}_{i}=\hat{n}\left[1+\frac{2(D-1)}{D-2} \phi_{0}\right], \\
& \sum_{i=1}^{D-1} \hat{p}_{i}^{2}=\hat{n}^{2}\left[\frac{1}{D-1}+\frac{K^{2}}{V_{0}^{2}}+\frac{4 \phi_{0}}{D-2}\left(1+\frac{D-1}{D-2} \phi_{0}\right)\right] .
\end{aligned}
$$

In the string frame the general physical behavior of the potential free dilatonic Bianchi type I universe is quite similar to that in the Einstein frame. The geometry is of the Kasner type, with a power-law type time dependence of the scale factors. The mean anisotropy of the space-time is constant and the Universe will never isotropize. On the other hand, if the condition $\hat{h}>D-1$ is fulfilled, the Universe ex-

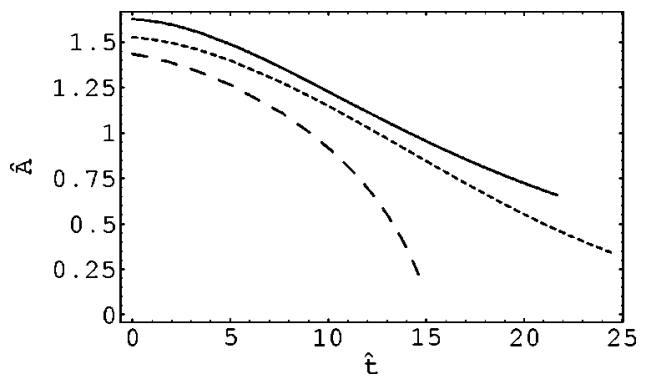

FIG. 6. Time variation of the anisotropy parameter $\hat{A}$ in the string frame for $\alpha=-1 / 3, \beta=1, \gamma=1$ and for different values of $\lambda$ : $\lambda=3$ (solid curve), $\lambda=2$ (this case corresponds to the presence of a central charge deficit or cosmological constant) (dotted curve) and $\lambda=1$ (dashed curve). We have used the normalization $(D-2) /(D-1) U_{0}=1$.

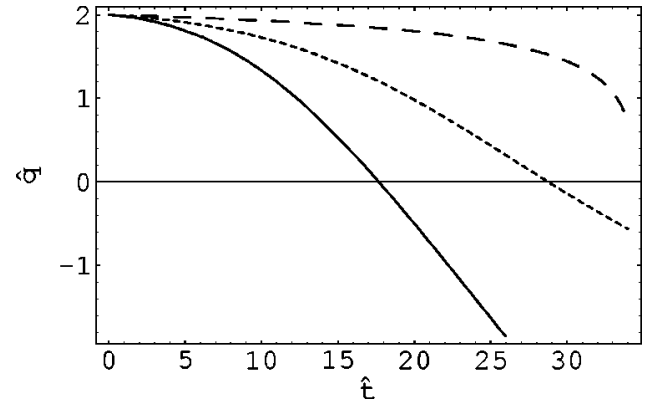

FIG. 7. Dynamics of the deceleration parameter $\hat{q}$ in the string frame in the presence of the exponential potential $\hat{U}=U_{0} \exp \{[\lambda$ $-4 /(D-2)] \phi\}, \quad \alpha=-1 / 3, \beta=1, \quad \gamma=1$ and $\lambda=3$ (solid curve), $\lambda=2$ (this case corresponds to the presence of a central charge deficit or cosmological constant) (dotted curve) and $\lambda=1$ (dashed curve). We have used the normalization $(D-2) /(D-1) U_{0}=1$.

periences an eternal power law type inflationary anisotropic phase. Hence in the string frame a dilaton field filled Bianchi type I universe provides an example of an inflating but never isotropizing cosmological type evolution.

In the string frame and in the presence of an exponential potential $\hat{U}(\phi)=\hat{U}_{0} \exp (\hat{\lambda} \phi)$, with $\hat{\lambda}=\lambda-4 /(D-2)$ and $\lambda$ an arbitrary constant, the Bianchi type I universe shows a very large variety of behaviors. In Figs. 5-8 we represented the dynamics of the volume scale factor, anisotropy parameter, deceleration parameter and potential for different values of $\lambda$ (different exponential potential functions) but for fixed $\alpha, \beta$ and $\gamma$. These solutions generically begin from a singular state, followed by an expansionary phase, with the volume scale factor and scale factors reaching a local maximum. Then the Universe re-collapses into a new singular phase. This type of evolution is associated with an initial rapid isotropization of the space-time, with the mean anisotropy parameter $\hat{A}$ rapidly decreasing. Near the second singular state the evolution of the Universe is generally inflationary, with the string frame deceleration parameter $\hat{q}$ smaller than zero, $\hat{q}<0$. After this phase the effect of the dilaton becomes irrelevant to the dynamics of space-time.

In the limit of large values of the parameter $u$, the term $-\alpha u^{2}$ dominates, $-\alpha u^{2} \gg \beta u-\gamma$. Hence in the limit of

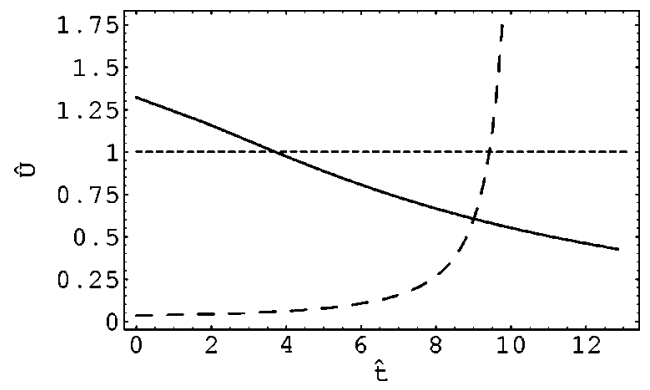

FIG. 8. Time evolution in the string frame of the exponential potential $\hat{U}=U_{0} \exp \{[\lambda-4 /(D-2)] \phi\}$ for $\alpha=-1 / 3, \beta=1, \gamma$ $=1$ and $\lambda=3$ (solid curve), $\lambda=2$ (this case corresponds to a constant potential $\hat{U}=$ const) (dotted curve) and $\lambda=1$ (dashed curve). We have used the normalization $(D-2) /(D-1) U_{0}=1$. 
large $u$ (and large time, $\hat{t} \rightarrow \infty$, too), from Eq. (33) we obtain $V \simeq V_{0} u^{-1 / \alpha}$. Therefore from Eq. (66) it follows that $\hat{t}$ $\simeq u^{(1+1 / \alpha)[4 /(D-2) \lambda-1]} \simeq u^{1 / \hat{l}}$ and, consequently,

$$
\begin{aligned}
& \hat{V} \simeq \hat{t}^{(D-1) \hat{l}^{\prime}}, \\
& \hat{H} \simeq \hat{t}^{-1}, \\
& \hat{a}_{i} \simeq \hat{t}^{\prime} \exp \left(\frac{V_{0} K_{i}}{\alpha} \hat{t}^{-\hat{l}}\right), \quad i=1, \ldots, D-1, \\
& \hat{A} \simeq \hat{t}^{-2 \hat{l}}, \\
& \hat{q} \simeq(D-2)+\frac{(D-1) \alpha \kappa^{2}}{V_{0}(\kappa-\lambda)^{2}}, \\
& \hat{U} \simeq \hat{t}^{-2},
\end{aligned}
$$

where we denoted

$$
\begin{aligned}
& \hat{l}=\frac{1}{\left(1+\frac{1}{\alpha}\right)\left[\frac{4}{(D-2) \lambda}-1\right]}, \\
& \hat{l}^{\prime}=1+\hat{l}\left[1+\frac{D-2}{(D-1) \alpha}\right] .
\end{aligned}
$$

In the long-time limit the behavior of the exponential potential dilaton field filled universe is quite different from the behavior of the potential free dilatonic anisotropic universe. The dependence of the coefficients $\hat{l}, \hat{l}^{\prime}$ on the two constants $\alpha$ and $\lambda$ leads to a larger spectrum of admissible final states, with isotropic inflationary or non-inflationary evolution or re-collapse into a singular state. For $|\alpha|<1$ generally $\hat{l}<0$, and, if $\hat{l}<-[1+(D-2) /(D-1) \alpha]^{-1}$, then in the string frame the volume scale factor tends to zero, $\hat{V} \rightarrow 0$.

The requirement that in the string frame the Universe inflate, $\hat{q}<0$, leads, with the use of Eq. (103), to the following inequality relating the coupling constant $\kappa$ to $\lambda$ :

$$
\kappa\left(\frac{\lambda}{\kappa}\right)^{2}+4 V_{0}\left(1-\frac{\lambda}{\kappa}\right)^{2}<4 \frac{D-1}{D-2} .
$$

This condition represents the generalization to the string frame of the Einstein frame condition (88). In the string frame the inflationary behavior sensitively depends not only on $\lambda$ and $\kappa$ but also on the integration constant $V_{0}$.

It is well known that the action (1) with vanishing antisymmetric field strength $H_{[3]}$ is invariant with respect to scale factor duality transformations of the form $G \rightarrow \bar{G}$ $=G^{-1}$ and $\phi \rightarrow \bar{\phi}-\ln (\operatorname{det} G)$, where $G$ is a matrix build from the metric tensor components of the FRW, anisotropic or inhomogeneous metric [10]. The inclusion of the potential breaks this duality, but leads, on the other hand, to the possibility of obtaining more general models allowing a better physical description of the very early evolution of our Universe.

\section{ACKNOWLEDGMENTS}

One of the authors (T.H.) would like to thank Dr. P. Blaga for useful suggestions. C.M.C. thanks Professor J. M. Nester for profitable discussions. The work of C.M.C. was supported in part by the National Science Council (Taiwan) under grant NSC 89-2112-M-008-016.
[1] M. Gasperini and G. Veneziano, Astropart. Phys. 1, 317 (1993).

[2] C. G. Callan, E. J. Martinec, M. J. Perry, and D. Friedan, Nucl. Phys. B262, 593 (1985).

[3] C. Lovelace, Nucl. Phys. B273, 413 (1986).

[4] L. J. Romans, Phys. Lett. 169B, 374 (1986).

[5] J. E. Lidsey, D. Wands, and E. J. Copeland, "Superstring Cosmology," hep-th/9909061.

[6] M. Gasperini and R. Ricci, Class. Quantum Grav. 12, 677 (1995).

[7] R. Brandenberger, R. Easther, and J. Main, J. High Energy Phys. 09, 007 (1998).

[8] A. L. Maroto and I. L. Shapiro, Phys. Lett. B 414, 34 (1997).

[9] R. Easther, K. Maeda, and D. Wands, Phys. Rev. D 53, 4247 (1996).

[10] E. Di Pietro and J. Demaret, Int. J. Mod. Phys. D 8, 349 (1999).

[11] A. Lukas, B. A. Ovrut, and D. Waldram, "The Cosmology of $M$-Theory and Type II Superstrings,' hep-th/9802041.

[12] M. Gasperini, "'Elementary Introduction to Pre-Big Bang Cosmology and to the Relic Graviton Background," hep-th/9907067.
[13] E. J. Copeland, A. Lahiri and D. Wands, Phys. Rev. D 50, 4868 (1994).

[14] A. Lukas, B. A. Ovrut, and D. Waldram, Nucl. Phys. B495, 365 (1997).

[15] G. F. R. Ellis, D. C. Roberts, D. Solomons, and P. K. S. Dunsby, "Using the Dilaton Potential to Obtain String Cosmology Solutions,"' gr-qc/9912005.

[16] L. C. Garcia de Andrade, Phys. Lett. B 468, 28 (1999).

[17] G. Veneziano, “A Simple/Short Introduction to Pre-Big Bang Physics/Cosmology," hep-th/9802057.

[18] J. D. Barrow, Phys. Lett. B 187, 12 (1987).

[19] A. B. Burd and J. D. Barrow, Nucl. Phys. B308, 929 (1988).

[20] J. E. Lidsey, Class. Quantum Grav. 9, 1239 (1992).

[21] J. M. Aguirregabiria, A. Feinstein, and J. Ibanez, Phys. Rev. D 48, 4662 (1993).

[22] J. M. Aguirregabiria, A. Feinstein, and J. Ibanez, Phys. Rev. D 48, 4669 (1993).

[23] A. Feinstein, J. Ibanez, and P. Labraga, J. Math. Phys. 36, 4962 (1995).

[24] L. P. Chimento and A. S. Jakubi, Int. J. Mod. Phys. D 5, 71 (1996). 
[25] J. Ibanez and I. Olasagasti, J. Math. Phys. 37, 6283 (1996).

[26] A. A. Coley, J. Ibanez, and R. J. van den Hoogen, J. Math. Phys. 38, 5256 (1997).

[27] E. J. Copeland, A. R. Liddle, and D. Wands, Phys. Rev. D 57, 4686 (1998).

[28] A. P. Billyard, A. A. Coley, and R. J. van den Hoogen, Phys. Rev. D 58, 123501 (1998).

[29] K. M. Mubarak and M. Oezer, Class. Quantum Grav. 15, 75 (1998).

[30] M. Susperregi and A. Mazumdar, Phys. Rev. D 58, 083512
(1998).

[31] J. Ibanez and I. Olasagasti, Class. Quantum Grav. 15, 1937 (1998).

[32] S. Byland and D. Scialom, Phys. Rev. D 57, 6065 (1998).

[33] A. A. Coley, "Dynamical Systems in Cosmology," gr-qc/9910074.

[34] B. de Carlos, J. A. Casas, and C. Munoz, Nucl. Phys. B399, 623 (1993).

[35] O. Gron, Phys. Rev. D 32, 2522 (1985). 